\title{
A STUDY ON NON VENEREAL GENITAL DERMATOSES IN NORTH INDIA
}

\author{
by Neerja Puri, Asha Puri
}

\author{
comment: \\ José Manuel Ríos-Yuil, M.D., MSc. \\ University of Panama and Panamanian Social Security, Panama \\ E-mail:jmriosyuil@hotmail.com
}

\author{
Source of Support: \\ Nil \\ Competing Interests: \\ None declared
}

Non-venereal genital dermatoses are a complicated group of illnesses because most of the patients that suffer from them feel embarrassed. They frequently think that all genital skin diseases are sexually transmitted, so they usually don't consult until the disease burden is unbearable. When that finally happens, some of these illnesses are already at an advanced stage that could endanger their lives. To make things worse, a lot of these patients get into serious troubles with their couples when they find about their disease.

Genital dermatoses are not easy to diagnose because their morphology is modified by the special environment of the genital area which produces heat, friction and occlusion [1]. For example, scales, a major finding in dermatoses like psoriasis, are less prominent than in other topographies. Fortunately, some of these dermatoses also affect other areas of the body, so examining extragenital areas aids in the diagnosis. A broad range of inflammatory, non-venereal dermatoses affect the genital skin. Some of them, like vitiligo, manifest as hypopigmented areas. But it is important to remember that lichen sclerosus (LS), lichen simplex chronicus and vulvar intraepithelial neoplasia (VIN) might also manifest as hypopigmented lesions [1]. Physicians should be aware of the diagnosis of LS, because this disease is associated with a risk of developing invasive squamous cell carcinoma (SCC) in both male (4-6\%) and female (2-5\%) patients [2,3]. Erythematous lesions of the genital area can be caused by tinea, candidiasis, psoriasis or dermatitis (atopic, contact or seborrheic). It is important not to underestimate the prevalence of contact dermatitis, especially the irritative type, which can be the primary cause of the patient's complaints or could be superimposed to other dermatoses [1-4]. Other entities that can manifest as erythematous or erythematosquamous plaques are Zoon's balanitis or vulvitis, VIN, extramammary Paget's disease, erythroplasia of Queyrat (EQ) and Bowen's disease (BD) [1-5]. Erosive genital lesions are not uncommon either. Lichen planus, pemphigus vulgaris, bullous pemphigoid, mucous membrane pemphigoid, linear IgA bullous disease, Hailey-Hailey disease, Darier disease, lupus erythematosus, Behçet disease, Crohn disease and SCC can cause erosive disease. In many cases, biopsy is the only way to make the final diagnosis [1-5]. It is worth mentioning that physicians should be well familiarized with the anatomy of the genital region, so normal findings such as penile pearly papules do not end being treated as genital warts [2].
It is interesting that this study didn't find cases of VIN or SCC in female patients or cases of EQ or BD in male patients. That could be explained, at least in part, because most of their patients were relatively young. It is well known that penile carcinoma in situ (EQ/BD) and SCC typically affect uncircumcised men older than 60 years $[2,4]$. The same is true for most of the cases of vulvar SCC and differentiated VIN, the subtype of VIN with the highest malignant potential [5]. It is worrisome to notice that older patients were not seeking attention in this study because they are the ones at the highest risk of developing neoplastic diseases that might put their lives in danger if not treated on time. Older patients are probably not coming because, as it happens worldwide, this age group tends to be less educated. This highlights the importance that patient education also has in the prevention and early diagnosis of genital dermatoses.

Even though the number of patients included was small, I think that this study is very important because it draws the attention of dermatologists and other physicians to a group of diseases that has been neglected. Genital dermatoses are usually neglected because dealing with them means dealing with a lot of psychosocial factors that are time consuming for physicians. Most dermatologists don't routinely ask about genital problems until they are expressly told by the patient and most Gynecologists and Urologists usually concentrate their attention into other areas of the genitourinary anatomy. This study will certainly serve as an incentive for other researchers to direct their attention to these dermatoses. All these factors might contribute to a better diagnosis and treatment of nonvenereal genital dermatoses.

\section{REFERENCES}

1. Drummond C: Common vulval dermatoses. Aust Fam Physician. 2011;40:490-6.

2. Teichman JM, Sea J, Thompson IM, Elston DM: Noninfectious penile lesions. Am Fam Physician. 2010;81:167-74.

3. Barchino-Ortiz L, Suárez-Fernández R, Lázaro-Ochaita P: [Vulvar Inflammatory Dermatoses]. Actas Dermosifiliogr. 2012;103:260-75. 4. Buechner SA: Common skin disorders of the penis. BJU Int. 2002;90:498-506.

5. Terlou A, Blok LJ, Helmerhorst TJ, van Beurden M: Premalignant epithelial disorders of the vulva: squamous vulvar intraepithelial neoplasia, vulvar Paget's disease and melanoma in situ. Acta Obstet Gynecol Scand. 2010;89:741-8. 